\title{
Spatiotemporal Dynamics of Tuberculosis Disease and Vaccination Impact in North Senatorial Zone Taraba State Nigeria
}

\author{
${ }^{1}$ A. A. Momoh, ${ }^{2}$ James, J. Yakoko, ${ }^{3}$ A. Tahir and ${ }^{4}$ Chukkol Y. B. \\ ${ }^{1,2,3,4}$ Department of Mathematics, Modibbo Adama University of Technology, P.M.B. 2076, Yola, Adamawa \\ State, Nigeria.
}

\begin{abstract}
In this work, The researchers present Passively Immune Infant $\left(V_{m}\right)$-Susceptible class $\left(S_{m}\right)$ Infection class $\left(I_{m}\right)$-Recovery class $\left(R_{m}\right)$ model to study the dynamic of tuberculosis transmission and vaccination impact in North Senatorial Zone, Taraba State, Nigeria. The compartment of the model is presented in a system of ordinary differential equations. Quantitative analysis of the model was done to investigate the equilibrium and stability of the model. An analytical approach was used to determine their Disease Free equilibrium and the Epidemic equilibrium state. The stability of the epidemic equilibrium is tested using Bellman and Cooke's theorem.

The model had two equilibrium position: The disease free equilibrium which was asymptotically stable for $R_{e}(\lambda)<0$ and the endemic equilibrium which was locally asymptotically stable for as it satisfies the Bellman and Cooke's condition for stability i.e. $|J|>0$.
\end{abstract}

Key words: Tuberculosis (TB), Vaccination, Infection, Equilibrium analysis, Stability analysis.

\section{Introduction}

Tuberculosis is mostly transmitted through air by infected persons coughing, sneezing or spiting with pulmonary tuberculosis. The probability of transmission per contact, per relevant unit time is general quite low. The bacteria may lodge in the person's lung and multiply. If the immune system in the lung is able to fight the bacteria and render it inactive (wall off) as a result of vaccination or treatment from $\mathrm{TB}$, the person will develop latent TB which is not infectious and cannot harm others.

Infected individuals may remain asymptomatic over their entire live (Latent TB). Active TB (the clinical disease) can develop into pulmonary and extra pulmonary form. Extra pulmonary TB is common in children while pulmonary TB is frequent in adult. Mycobacterium tuberculosis, the casual agent of the disease is transmitted almost exclusively via pulmonary cases. Cases arising within Five (5) years from first infection are classified as primary tuberculosis while cases arising after five (5) years from first infection are known as secondary tuberculosis. The asymptomatic individual may remain at latent for a long period of time due to vaccine durations and the antibody defense deposit in the body unless cured by treatment with antibiotics such as Ethanbutol, Rifanpacine, B6 e.t.c. if the bacteria later become active due to breakdown of the immune system, the person is symptomatic and infectious and can be cured (recovered) if treated otherwise die from the infection.

The person when cured becomes susceptible as he is likely to be re-infected on contact with an infected person. Recent research efforts have been geared towards studying the heterogeneous factors in the population with a view to incorporate them into the model. The efforts that have been made by researchers and their findings on various aspects of TB disease dynamics falls under the following subheadings.

i. Transmission

ii. Vaccination against tuberculosis

iii. Protective efficacy of the vaccine and duration of immunity

iv. Infection

v. Treatment

vi. Prevention

Most early transmission models were deterministic but recent resurgence of tuberculosis (TB) in develop countries and increase in cases of casualty contact and public transport suggested a model based on thorough understanding of the dynamics of the disease.

Castillo-Chavez and Feng (1998) also consider an age-structured model, in this case with agedependent transmission rates. They include one form of latency, as in their earlier work, and this model does not 
contain re-infection. They study vaccination, and define a vaccine-dependent $R_{0}$ threshold $\mathrm{R}(\psi)$. They prove stability of the disease-free equilibrium when $\mathrm{R}(\psi)<1$, and the existence of an endemic steady state when $\mathrm{R}($ $\psi$ ) $>1$, and discuss analytically-determined optimal vaccination strategies. These turn out to be either vaccination at a single age, or vaccination in precisely two age classes.

Aparacio et al (2000) develop a generalized household model, which took close and casual contact into account. The house cluster comprises of social networks (Family member, office mates, classmates, any person who have prolong contact with an active case). The basic reproductive number for the model is

$$
R_{o}=\frac{\beta_{n} k}{\beta+\gamma+\mu+k}
$$

Where $\beta$ the transmission rate, $\mathrm{n}$ is the size of the cluster, $\mu$ natural mortality rate, $\gamma$ the total percapita removal rate from infected and $\mathrm{k}$ is the progression rate to active TB. BCG-induced immunity develops about six weeks after vaccination. Experimental studies indicate that the mechanism of protection by BCG vaccination consists in reduction of the hematogenous spread of bacilli from the site of primary infection Smith \& Harding (1979) mediated by memory T lymphocytes induced by the first exposure to BCG. There is no evidence that BCG reduces the risk of becoming infected with tuberculosis bacilli, but it prevents forms of tuberculosis depending on hematogenous spread of the bacillus Heimbeck (1929). This inhibition of the hematogenous spread of bacilli thus reduces the risk of immediate disease and of disease due to reactivation. Because there is reduction in risk of immediate disease, but not of infection, there is a difference in the protective effect of BCG, depending on the type of tuberculosis infection. Myint et al. (1987).

The best method for determining the protective efficacy of a vaccine is a prospective, randomized, double-blind, placebo-controlled trial. These studies are difficult and expensive, and have rarely been performed. WHO has recently sponsored studies to evaluate the protective efficacy of BCG immunization in infants and/or children by two low-cost methods: case-control studies Smith $(1982,1987)$ and contact studies Ten Dam (1987).

These studies have recently been reviewed by Milstien and Gibson (1989), who concluded that there is good evidence that the efficacy of modern BCG vaccines is in the range of $60 \%$ to $90 \%$ for disseminated tuberculosis or meningitis in young children, but somewhat lower for other forms of primary tuberculosis disease. They also found no evidence that one BCG preparation tested was more efficacious than any other under the conditions of the trials, and thus no evidence to support the choice of one preparation or manufacturer of BCG over another on the basis of protective efficacy. A recent matched case-control study in Bangkok Sirinavin et al. (1991) found an adjusted protective efficacy for neonatal BCG vaccination of 83\%, and provided evidence to support the thesis that the accuracy of tuberculosis diagnosis, the types of tuberculosis, the length of time after vaccination, and the household tuberculosis exposure contribute to variation in the reported protective efficacy of neonatal BCG vaccination. There is a need for the development of a single in vitro test capable of predicting the induction of immune resistance of humans to infection or dissemination of M. tuberculosis. Despite the difficulty in interpretation of data, several trials have shown that efficacy of BCG is highest among the youngest recipients, and that it falls with increasing age at vaccination Fine et al. Tuberculosis Prevention Trial (1979).

It is therefore difficult to assess the impact of BCG vaccination programmes. However, when BCG immunization of newborns was stopped in Sweden, the incidence of the disease in infants raised six fold Romanus (1987). There are several publications which suggest that the protection BCG provides against tuberculosis is a function of the relative importance of disease due to endogenous reactivation as compared to re infection from the outside. Thus, BCG protects against hematogenous spread of infection Fine (1988), Ten Dam (1984). This suggestion predicts the greater protective efficacy seen against miliary tuberculosis and tuberulous meningitis, compared with pulmonary tuberculosis Smith (1987).

\section{Prevention and Control for Tuberculosis}

Dye et al (1998) also have developed an age-structured model, with the goal of exploring TB control under the DOTS strategy and under improved case detection and cure. Their model uses discrete time rather than a partial differential equation framework. It includes two latent classes (slow and fast) with a set portion of new infections moving into each, as well as re-infection. They validate their model by comparing their results to annual risk of infection data from the Netherland. They then simulate different epidemiological situations. They conclude that if tuberculosis is stable, and if HIV is not included in the model, then WHO targets of $70 \%$ case detection and $85 \%$ cure "would reduce the incidence rate by $11 \%$ (range $8-12$ ) per year and the death rate by $12 \%$ (9-13) per year". The effect would be smaller if TB were already in decline. They conclude that DOTS has greater potential today (in developing countries) than it did 50 years ago in developed countries, but that case detection and cure rates must be improved. 
Salomon et al. (2006) extend this model, calibrating it to natural TB epidemics and then studying the effects of hypothetical new treatments with differing durations. In their model, drug resistance could result from transmission and acquisition. They first calibrate the model to represent TB in South-East Asia. They conclude that if more rapid treatments for TB were available, with reduced infectious periods, this could significantly reduce TB mortality and incidence, particularly if these become available soon.

There are, of course, other alternatively-structured models. Salpeter and Salpeter (1998) used TB incidence data to derive a function for the time delay from initial infection to active disease, and develop an integral equation for $R_{0}$. This is not a dynamic epidemiological model for TB, but it is relevant to TB models because the variable delays and widely ranging estimates of $R_{0}$ are important. They report $R_{0}$ near 1 , which was associated with time scales of approximately 100 years.

\section{Model Formulation}

The model is going to divided into four classes of population under study namely;

i. The passively immune infant

ii. Susceptible Class

iii. Infected Class

Iv. Recovery Class

Based on this, the researchers divided the populations into four compartments represented as:

\section{Model Diagram}

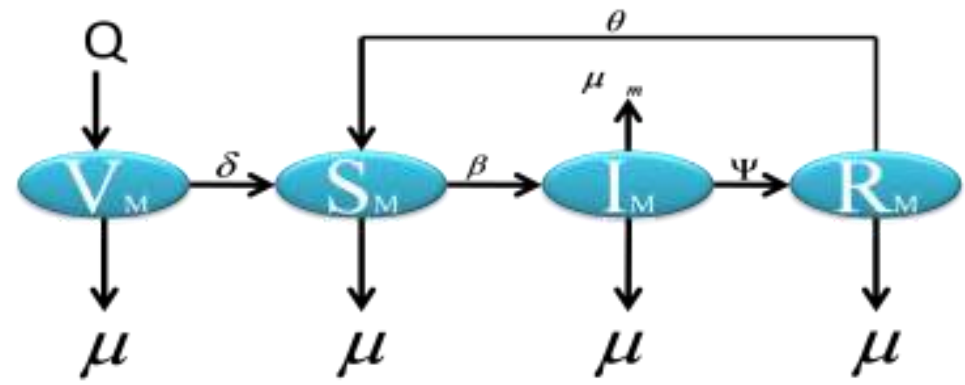

Figure 1: Schematic representation of VSIR Model

Individuals are recruited into $V_{m}$ class via natural birth at the rate $Q$ through passive vaccination, the population of the $V_{m}$ decreases due to natural death at rate $\mu$ and movement of the individuals into $S_{m}$ as a result of warning out of vaccines used in passive vaccination at the rate $\delta$. The population of the $S_{m}$ increases due to coming of individuals from the $V_{m}$ and $R_{m}$ classes at the rates $\delta$ and $\boldsymbol{\theta}$. The population of the $S_{m}$ class decreases due to the movement of individuals into the infected class at the rate $\beta$ and natural death at a rate $\boldsymbol{\mu}$. The population of the $\boldsymbol{I}_{m}$ decreases due to treatment against TB at the rate $\boldsymbol{\psi}$ and natural death at the rate $\boldsymbol{\mu}$ and death as result of TB infection at the rate $\boldsymbol{\mu}_{m}$. The population of the $\boldsymbol{R}_{m}$ increases due to movement of individuals at the rates $\boldsymbol{W}$ from $\boldsymbol{I}_{m}$ and decreases due to movement of individuals into the $S_{m}$ at the rate $\boldsymbol{\theta}$ and natural death at rate $\boldsymbol{\mu}$

\section{Definition of parameters}

$\mathrm{Q}=$ Natural Birth rate

$V_{m}=$ Passively immune infant at the time $\mathrm{t}$

$S_{m}=$ Susceptible class at the time $\mathrm{t}$

$\boldsymbol{I}_{m}=$ Infected class at time t

$\boldsymbol{R}_{m}=$ Removed class at time $\mathrm{t}$

$\boldsymbol{\mu}=$ Natural death rate

$\delta=$ Rate of the duration of vaccine efficacy

$\boldsymbol{\beta}=$ TB contact rate 
$\boldsymbol{\mu}_{m}=$ Death as a result of $\mathrm{TB}$ infection

$\psi=$ Rate of recovery from $\mathrm{TB}$ infection

$\boldsymbol{\theta}=$ Rate at which individuals become susceptible

Model Equation

The model above is thus described by the following set of ordinary differential equations

$$
\begin{aligned}
& \frac{d V_{m}}{d t}=Q-(\mu+\delta) V_{m} \\
& \frac{d S_{m}}{d t}=\delta V_{m}-\left(\mu+\beta I_{m}\right) S_{m}+\theta S_{m} \\
& \frac{d I_{m}}{d t}=\beta S_{m} I_{m}-\left(\mu+\mu_{m}+\psi\right) I_{m} \\
& \frac{d R_{m}}{d t}=\psi I_{m}-(\mu+\theta) R_{m}
\end{aligned}
$$

IV. Equilibrium State of the Model

Let $\quad V_{m}=X_{1}, \quad S_{m}=X_{2}, \quad I_{m}=X_{3}, \quad R_{m}=X_{4}$

Equations 1-4 becomes,

$$
\begin{aligned}
& \frac{d X_{1}}{d t}=Q-(\mu+\delta) X_{1} \\
& \frac{d X_{2}}{d t}=\delta X_{1}-\left(\mu+\beta X_{3}-\theta\right) X_{2} \\
& \frac{d X_{3}}{d t}=\beta X_{2} X_{3}-\left(\mu+\mu_{m}+\psi\right) X_{3} \\
& \frac{d X_{4}}{d t}=\psi X_{3}-(\mu+\theta) X_{4}
\end{aligned}
$$

\section{Disease Free Equilibrium (DFE)}

From (5)

$$
\begin{aligned}
& \frac{d X_{1}}{d t}=Q-(\mu+\delta) X_{1} \\
& \Rightarrow Q-(\mu+\delta) X_{1}=0 \\
& \therefore X_{1}=\frac{Q}{(\mu+\delta)}
\end{aligned}
$$

From (6)

$$
\begin{aligned}
& \Rightarrow \frac{d X_{2}}{d t}=\delta X_{1}-\left(\mu+\beta X_{3}-\theta\right) X_{2} \\
& \Rightarrow \delta X_{1}-\left(\mu+\beta X_{3}-\theta\right) X_{2}=0 \\
& \therefore X_{2}=\frac{\delta X_{1}}{\left(\mu+\beta X_{3}-\theta\right)}
\end{aligned}
$$

Substituting (9) into (10) we have, 


$$
\begin{aligned}
& \Rightarrow X_{2}=\frac{\delta\left(\frac{Q}{(\mu+\delta)}\right)}{\left(\mu+\beta X_{3}-\theta\right)} \\
& \therefore X_{2}=\frac{\delta Q}{(\mu+\delta)\left(\mu+\beta X_{3}-\theta\right)} \\
& X_{2}=\frac{\delta Q}{(\mu+\delta)(\mu-\theta)}
\end{aligned}
$$

From (7)

$$
\begin{aligned}
& \Rightarrow \frac{d X_{3}}{d t}=\beta X_{2} X_{3}-\left(\mu+\mu_{m}+\psi\right) X_{3} \\
& \Rightarrow \beta X_{2} X_{3}-\left(\mu+\mu_{m}+\psi\right) X_{3}=0 \\
& {\left[\beta X_{2}-\left(\mu+\mu_{m}+\psi\right)\right] X_{3}=0}
\end{aligned}
$$

Therefore, either,

$$
X_{3}=0
$$

OR

$$
\begin{aligned}
& \Rightarrow \beta X_{2}-\left(\mu+\mu_{m}+\psi\right)=0 \\
& X_{2}=\frac{\left(\mu+\mu_{m}+\psi\right)}{\beta}
\end{aligned}
$$

Substituting (12) into (10) gives,

$$
\therefore X_{2}=\frac{\delta Q}{(\mu+\delta)(\mu-\theta)}
$$

Substituting (12) into (8) gives,

$$
X_{4}=\mathrm{O}
$$

Hence, the disease free equilibrium (DFE) states are as follows

$$
\left(X_{1}, X_{2}, X_{3}, X_{4}\right)=\left(\frac{Q}{(\mu+\delta)}, \frac{\delta Q}{(\mu+\delta)(\mu-\theta)}, 0,0\right)
$$

\section{Epidemic Equilibrium State (EE)}

Substituting (8) into (11)

$$
\therefore X_{3}=\frac{\delta Q}{(\mu+\delta)\left(\mu+\mu_{m}+\psi\right)}-\frac{(\mu-\theta)}{\beta}
$$

Recall from (8)

$$
\begin{aligned}
& \Rightarrow \psi X_{3}-(\mu+\theta) X_{4}=0 \\
& X_{4}=\frac{\psi X_{3}}{(\mu+\theta)}
\end{aligned}
$$

Substituting (13) into (14)

$$
X_{4}=\frac{\psi \delta Q}{(\mu+\delta)(\mu+\theta)\left(\mu+\mu_{m}+\psi\right)}-\frac{\psi(\mu+\theta)}{\beta(\mu+\theta)}
$$

17

Hence, the Epidemic Equilibrium (EE) states are as follows:

$$
X_{1}=\frac{Q}{(\mu+\delta)}
$$




$$
\begin{aligned}
& X_{2}=\frac{\left(\mu+\mu_{m}+\psi\right)}{\beta} \\
& \therefore X_{3}=\frac{\delta Q}{(\mu+\delta)\left(\mu+\mu_{m}+\psi\right)}-\frac{(\mu-\theta)}{\beta} \\
& X_{4}=\frac{\psi \delta Q}{(\mu+\delta)(\mu+\theta)\left(\mu+\mu_{m}+\psi\right)}-\frac{\psi(\mu+\theta)}{\beta(\mu+\theta)}
\end{aligned}
$$

\section{Stability Analysis}

$$
|J|=\left[\begin{array}{cccc}
-(\mu+\delta) & 0 & 0 & 0 \\
\delta & -\left(\mu+\beta X_{3}-\theta\right) & -\beta & 0 \\
0 & \beta X_{3} & \beta X_{2}-\left(\mu+\mu_{m}+\psi\right) & 0 \\
0 & 0 & \psi & -(\mu+\theta)
\end{array}\right]
$$

$-(\mu+\delta)-\lambda\left(-\left(\mu+\beta X_{3}-\theta\right)-\lambda\left[\begin{array}{cc}\beta X_{2}-\left(\mu+\mu_{m}+\psi\right)-\lambda & 0 \\ \psi & -(\mu+\theta)-\lambda\end{array}\right]+\beta\left[\begin{array}{cc}\beta X_{3} & 0 \\ 0 & -(\mu+\theta)-\lambda\end{array}\right]\right)=0$

$-(\mu+\delta)-\lambda\left(-\left(\mu+\beta X_{3}-\theta\right)-\lambda\right)\left(\beta X_{2}-\left(\mu+\mu_{m}+\psi\right)-\lambda\right)(-(\mu+\theta)-\lambda)+\left[\beta\left(-\beta X_{3}(\mu+\theta)-\lambda\right)\right]=0$

$\left.-(\mu+\delta)-\lambda\left(-\left(\mu+\beta X_{3}-\theta\right)-\lambda\right)\left[\left(\beta X_{2}-\left(\mu+\mu_{m}+\psi\right)-\lambda\right)+\beta^{2} X_{3}\right)(-(\mu+\theta)-\lambda)\right]=0$

\section{Disease Free Stability Analysis}

The characteristic equation above takes the form:

$\left.-(\mu+\delta)-\lambda\left(-\left(\mu+\beta X_{3}-\theta\right)-\lambda\right)\left[\left(\beta X_{2}-\left(\mu+\mu_{m}+\psi\right)-\lambda\right)+\beta^{2} X_{3}\right)(-(\mu+\theta)-\lambda)\right]=0$

$\Rightarrow$ Either,

$$
\therefore \lambda_{1}=-(\mu+\delta)
$$

OR

$$
\therefore \lambda_{2}=-(\mu-\theta)
$$

OR

$$
\begin{aligned}
& \lambda_{4}=\beta X_{2}-\left(\mu+\mu_{m}+\psi\right) \\
& \lambda_{4}=\frac{\beta \delta Q}{(\mu+\delta)(\mu-\theta)}-\left(\mu+\mu_{m}+\psi\right) \\
& \lambda_{4}=\frac{\beta \delta Q}{(\mu+\delta)(\mu-\theta)}<\left(\mu+\mu_{m}+\psi\right)
\end{aligned}
$$

This implies the for $\lambda_{4}$ to be asymptotically stable, $\left(\frac{\beta \delta Q}{(\mu+\delta)(\mu-\theta)}<\left(\mu+\mu_{m}+\psi\right)_{\text {where }}\right.$ $\frac{\beta \delta Q}{(\mu+\delta)(\mu-\theta)}$ is the number of susceptible individuals produced.

OR

$$
\lambda_{3}=-(\mu+\theta)
$$

Since then $\mathrm{R}_{e}\left(\lambda_{1}\right)<0, \mathrm{R}_{e}\left(\lambda_{2}\right)<0, \mathrm{R}_{e}\left(\lambda_{3}\right)<0, \mathrm{R}_{e}\left(\lambda_{4}\right)<0$, then it is asymptomatically stable. 


\section{Stability Analysis of the Epidemic Equilibrium}

When applying Bellman and Cooke's (1963), the result of Bellman and Cooke's theorem state that, if $\Delta(i y), y \varepsilon I$ is separated into real and imaginary parts $\Delta(i y)=F(y)+i G(y)$ and all zeros of $\Delta(z)$ have negative real parts then the zeros of $F(y)$ and $G(y)$ are real and alternative then:

$$
F(0) G^{\prime}(0)-F^{\prime}(0) G(0)>0
$$

(23) is applied to the characteristic equation (18). We consider (18) in the form $H(\lambda)=0$

And we obtain:

$$
\left.H(\lambda)=-(\mu+\delta)-\lambda\left(-\left(\mu+\beta X_{3}-\theta\right)-\lambda\right)\left[\left(\beta X_{2}-\left(\mu+\mu_{m}+\psi\right)-\lambda\right)+\beta^{2} X_{3}\right]-(\mu+\theta)-\lambda\right)
$$

Also, by applying Bellman and Cooke's theorem on above, setting $\lambda=i w$, we have $H(i w)=F(w)+i G(w)$

Where $F(w)$ and $i G(w)$ are real and imaginary part of $H(i w)$.

Substituting $\lambda=i w$ (24) to have

$$
\begin{aligned}
& \left.H(i w)=-(\mu+\delta)-i w\left(-\left(\mu+\beta X_{3}-\theta\right)-i w\right)\left[\left(\beta X_{2}-\left(\mu+\mu_{m}+\psi\right)-i w\right)+\beta^{2} X_{3}\right]-(\mu+\theta)-i w\right) \\
& \therefore F(w)=(\mu+\delta)\left(\mu+\beta X_{3}-\theta\right)-(\mu+\theta)\left[\beta X_{2}-\left(\mu+\mu_{m}+\psi\right)+\beta^{2} X_{3}\right]+2 w^{2} \\
& G(w)=w\left[(\mu+\delta)+\left(\mu+\beta X_{3}-\theta\right)+(\mu+\theta)-\beta X_{2}+\left(\mu+\mu_{m}+\psi\right)-\beta^{2} X_{3}\right] \\
& F^{\prime}(w)=4 w \\
& G^{\prime}(w)=(\mu+\delta)+\left(\mu+\beta X_{3}-\theta\right)+(\mu+\theta)-\beta X_{2}+\left(\mu+\mu_{m}+\psi\right)-\beta^{2} X_{3}
\end{aligned}
$$

Setting $w=0$

$$
F(0)=(\mu+\delta)\left(\mu+\beta X_{3}-\theta\right)-(\mu+\theta)\left[\beta X_{2}-\left(\mu+\mu_{m}+\psi\right)+\beta^{2} X_{3}\right]
$$

Substituting the values of $\mathrm{X}_{2}$ and $\mathrm{X}_{3}$ into $F(0)=0 \quad$ we have,

$$
\begin{aligned}
& F(0)=2 \mu^{2}-\left(2 \mu+\delta-\mu_{m}-\psi\right) \theta+\mu \mu_{m}+\mu \psi+\frac{\delta Q \beta(\mu+\delta-\beta(\mu+\theta))}{\left(\mu+\mu_{m}+\psi\right)(\mu+\delta)}-(\mu-\theta)[(\mu+\delta)+\beta(\mu+\theta)]-(\mu+\theta)\left(\mu+\mu_{m}+\psi\right) \\
& F^{\prime}(0)=0 \\
& G^{\prime}(0)=(\mu+\delta)+\left(\mu+\beta X_{3}-\theta\right)+(\mu+\theta)-\beta X_{2}+\left(\mu+\mu_{m}+\psi\right)-\beta^{2} X_{3}
\end{aligned}
$$

Substituting the values of $\mathrm{X}_{2}$ and $\mathrm{X}_{3}$ into $G^{\prime}(0)=0 \quad$ we have,

$$
G^{\prime}(0)=3 \mu+\delta+\psi+\mu_{m}-(1-\beta)(\mu-\theta)+\frac{\left(\beta-\beta^{2}\right) \delta Q}{(\mu+\delta)\left(\mu+\mu_{m}+\psi\right)}
$$

$G(0)=0$

We have from Bellman and Cooke's theorem (1963) that:

The condition $\mathrm{R}_{e}(\lambda)<0$; is given by the inequality:

$$
F(0) G^{\prime}(0)-F^{\prime}(0) G(0)>0
$$

The equation (29) is the stability condition for the Epidemic Equilibrium. But from (26) and (27) we have $F^{\prime}(0)=0$ and $G(0)=0$ 
Spatiotemporal Dynamics Of Tuberculosis Disease And Vaccination Impact In North Senatorial Zone

Hence, equation (29) becomes

$F(0) G^{\prime}(0)>0$

Let $|J|=F(0) G^{\prime}(0)$

Then the Epidemic equilibrium state is stable when $J>0$.

\section{Results and Discussion}

Data collected from Tuberculosis and Leprosy unit, Ministry of Health, Taraba state, Nigeria, was used to obtain the simulation for the model.

Using hypothetical values pertinent to Taraba State, the researchers obtain the following results using Maple software as shown below:

\begin{tabular}{|l|l|l|l|l|l|l|l|l|}
\hline $\mathrm{Q}$ & $\beta$ & $\theta$ & $\mu$ & $\Psi$ & $\delta$ & $\boldsymbol{\mu}_{m}$ & $\boldsymbol{J}$ & REMARKS \\
\hline 0.03923 & 0.1 & 0.5 & 0.01433 & 1.0 & 0.02 & 0.15 & 0.0699087 & STABLE \\
\hline 0.03923 & 0.2 & 0.5 & 0.01433 & 0.9 & 0.02 & 0.15 & 0.0688880 & STABLE \\
\hline 0.03923 & 0.3 & 0.5 & 0.01433 & 0.8 & 0.02 & 0.15 & 0.0666962 & STABLE \\
\hline 0.03923 & 0.4 & 0.5 & 0.01433 & 0.7 & 0.02 & 0.15 & 0.0633377 & STABLE \\
\hline 0.03923 & 0.5 & 0.5 & 0.01433 & 0.6 & 0.02 & 0.15 & 0.0587936 & STABLE \\
\hline 0.03923 & 0.6 & 0.5 & 0.01433 & 0.5 & 0.02 & 0.15 & 0.0530335 & STABLE \\
\hline 0.03923 & 0.7 & 0.5 & 0.01433 & 0.4 & 0.02 & 0.15 & 0.0460057 & STABLE \\
\hline 0.03923 & 0.8 & 0.5 & 0.01433 & 0.3 & 0.02 & 0.15 & 0.0376138 & STABLE \\
\hline 0.03923 & 0.9 & 0.5 & 0.01433 & 0.2 & 0.02 & 0.15 & 0.0143337 & STABLE \\
\hline 0.03923 & 1.0 & 0.5 & 0.01433 & 0.1 & 0.02 & 0.15 & 0.0156232 & STABLE \\
\hline 0.03923 & 1.1 & 0.5 & 0.01433 & 0.0 & 0.02 & 0.15 & -0.0002277 & UNSTABLE \\
\hline
\end{tabular}

Figure 2: Result of stability analysis of Epidemic Equilibrium State.

\section{Simulations}

The first example is concern with the estimation properties of the VSIR model given by equation (5) (8) in the presence of low contraction, while the second example is concern with the estimation properties of the VSIR model given by equation (5) - (8) in the presence of high contraction. The VSIR model is described by the following parameters taken from TB outbreak in North Senatorial zone of Taraba state. $\mathrm{Q}=0.03923, \beta=0.1$ $\& 0.01, \mu=0.01433, \mu_{m}=0.15, \theta=0.5, \delta=0.02, \psi=0.23$. The initial conditions are given by $V(0)$ $=419,359, S(0)=838,712, I(0)=536, R(0)=427$.

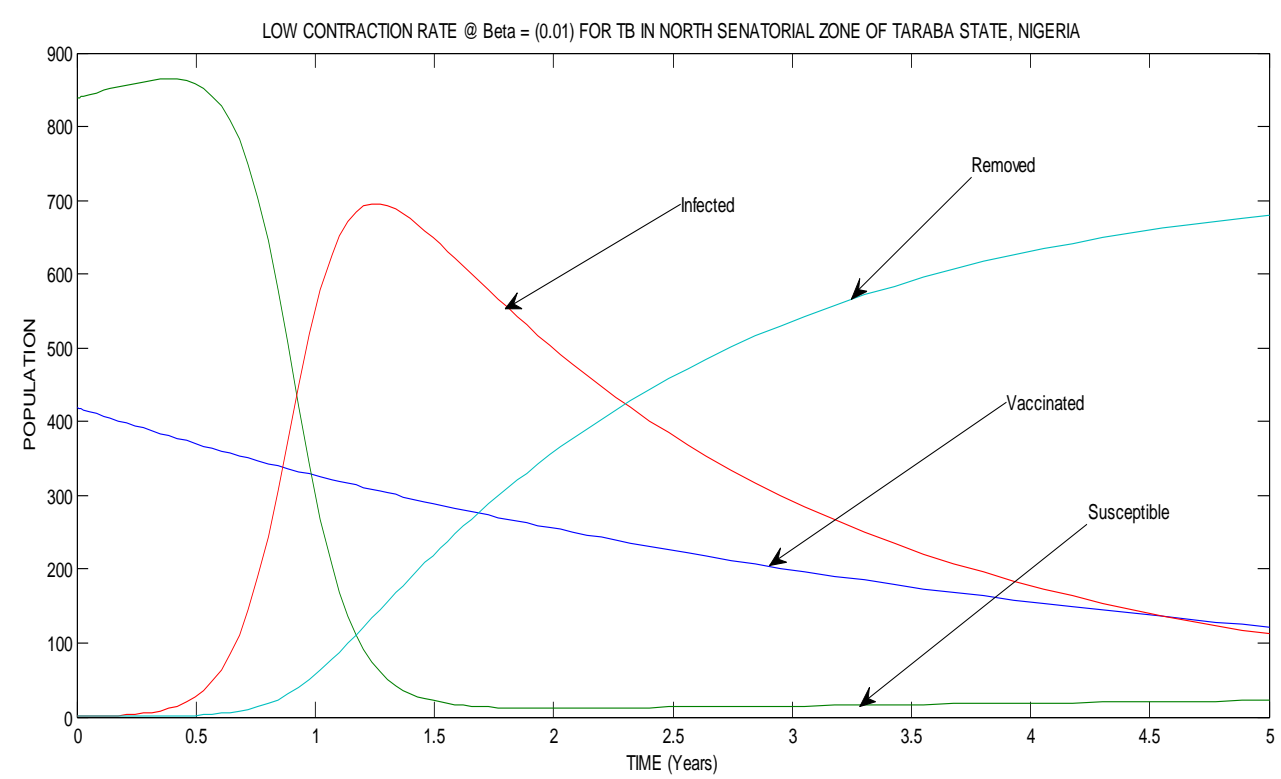

Figure 3: Graphical profile showing low contraction rate 


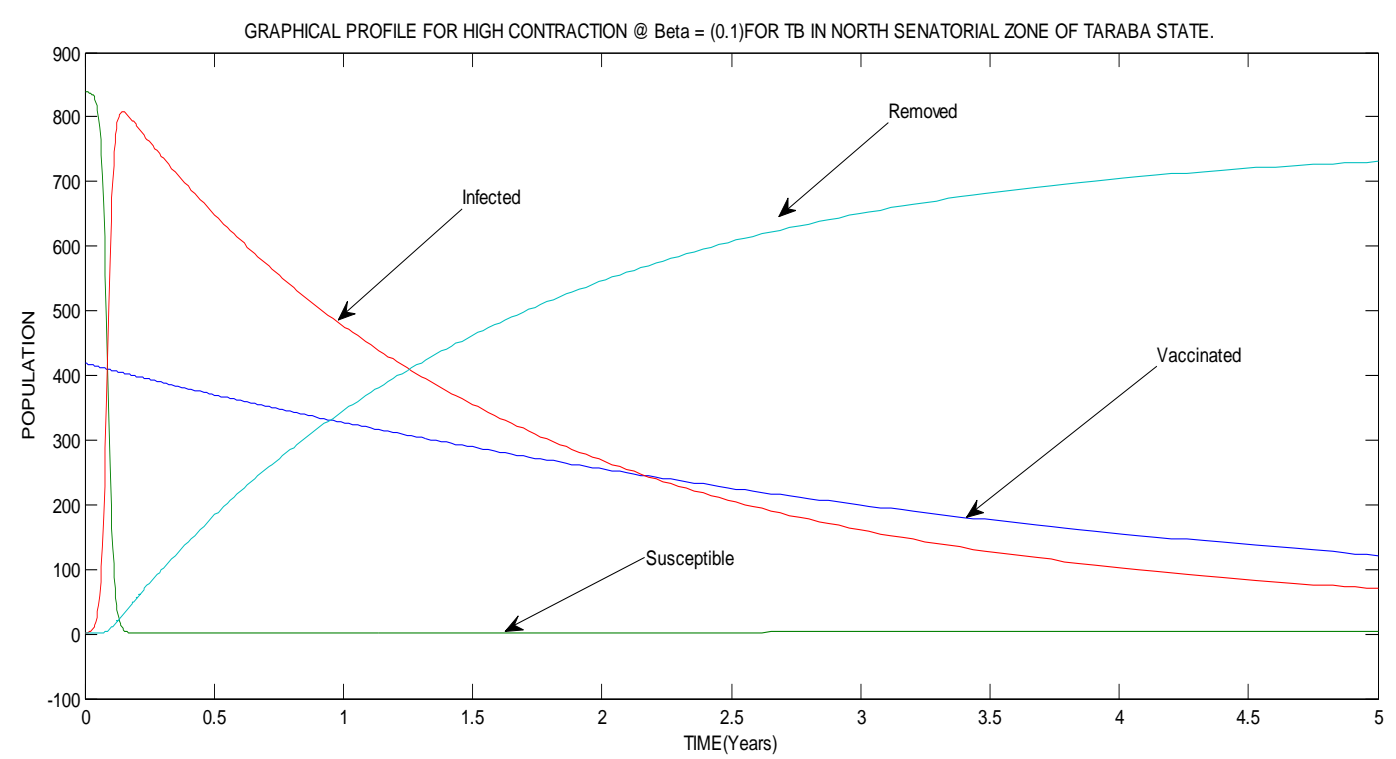

Figure 4: Graphical profile showing high contraction rate

Figure 3 shows the graphical profile of the VSIR model for $\beta=0.01$ (low contraction rate). It was observed that the population of the infected class increases steadily until when $T=1.3$, then it decreases steadily. While figure 4 shows the graphical profile of the VSIR model for $\beta=0.1$ (high contraction rate). It was observed that the population of the infected class increase exponentially until $T=0.25$ then, decreases steadily.

\section{Conclusion}

Conclusively, we will like to conclude that, Progression to active TB among the population is epidemiologically significant and interventions should focus on both vaccinations and treatment of infected persons, therefore, effort should be made to minimize the contraction rate for the sustenance of North Senatorial Zone of Taraba state. Anti-tuberculosis, treatment of adults is crucial in controlling the epidemic and intervention measures such as frequent vaccination and adhering to TB treatment should be proposed and implemented, they should target progression to active TB for those infected.

\section{Recommendations:}

Tuberculosis is an airborne disease and therefore prevention and control of tuberculosis could be achieved if the following measures are adopted:

1. Ensure good, adequate, hygiene measures of health are put in place.

2. Preventive vaccines such as Becillus Calmette Guerin (BCG) should be administered to particularly children and pregnant mothers.

3. All health care centres involve in TB treatment should adopt the Direct Observation Treatment short course (DOTS) strategy for treatment of patients.

4. Government should create awareness and sensitize the public on transmission and spread of TB.

5. Prevent or control exposure to infected animals and infected persons.

6. Government should embark on contact tracing for newly infected individuals, so that they can be placed on TB treatment therapy immediately.

\section{Reference}

[1] Aparicio, J.P., Capurri, A.F., Castillo-Chavez, C. (2000), “Transmission and dynamics of Tuberculosis on generalized household', J. Theoretical Biology.

[2] Bellman R. and Cooke K.C. (1963), "Differential difference equation”. London. Academic press.

[3] Castillo-Chavez, C. and Feng, Z., (1998), Global stability of an age-structure model for TB and its applications to optimal vaccination strategies. Math Biosci,151(2):135-154.

[4] Dye, C., Garnett, G. P., Sleeman, K. and Williams, B. G.(1998), Prospects for Worldwide tuberculosis control under the who dots strategy. Directly observed short-course therapy.

[5] Fine PEM (1988). BCG vaccination against tuberculosis and leprosy.Br Med Bull; 44:691-703.

[6] Fine PEM, Ponnighaus J.M., Maine N.P. (1986), The relationship between delayed type hypersensitivity and protective immunity induced by mycobacterial vaccines in man.Symposium on the Immunology of Leprosy, Oslo, Norway. 
[7] Heimbeck J. Sur la vaccination préventive de la tuberculose par injection sous-cutanée de BCG chez les élèves infirmières de l’hôpital Ulleval à Oslo (Norvège).

[8] Milstien JB, Gibson JJ. (1989)., Quality control of BCG vaccines by the World Health Organization: a review of factors that may influence vaccine effectiveness and safety. Bull WHO; 68:93-108.

[9] Myint TT, Win H, Aye HI-I, (1987), Case-control study on evaluation of BCG vaccination of newborn in Rangoon, Burma. Ann Trop Paediatr 1987;7:159-166.

[10] Ndaman I.(2010) A deterministic mathematical model of Tuberculosis disease dynamics, M. TECH thesis, F.U.T Minna.

[11] Romanus V. (1987), Tuberculosis in bacillus Calmette-Guerin immunized and non immunized children in Sweden: a tenyear evaluation following cessation of general bacillus Calmette-Guerin immunization of the newborn in 1975.

[12] Salomon, A. (2006), Prospects for advancing tuberculosis control efforts through novel therapies. PLoS Med, 3(8):e273,10.1371/journal.pmed.0030273

[13] Salpeter, E. E. and Salpeter, S. R. (1998), Mathematical model for the epidemiology of tuberculosis, with estimates of the reproductive number and infection delay function. Am J Epidemiol, 147(4):398-406.

[14] Sirinavin S, Chotpitayasunondh T, Suwanjutha S,(1991). Protective efficacy of neonatal Bacillus Calmette-Guerin vaccination against tuberculosis. Pediatric Infect Dis J1991;10:359-365.

[15] Smith P.G.(1982) Case-control studies of the efficacy of BCG against tuberculosis, In: Proc XXXV Work Conf Tub Resp Dis, Singapore. $1^{\text {st }}$ Edition.

[16] Smith P.G.(1987) Case-control studies of the efficacy of BCG against tuberculosis, In: Proc XXXV Work Conf Tub Resp Dis, Singapore. 2nd Edition.

[17] Smith, D.W., Harding, G.E.(1979), Are current strains of BCG protective in animals? Document TRI/SCG/79.6.1. Geneva: World Health Organization, 1979.

[18] Ten Dam H. G.(1987), Contact studies on the effectiveness of BCG vaccination in children. In: Proc XXXV Work Conf Tub Resp Dis, Singapore, 1986. Tokyo. 